\title{
Analysis and estimation of multi-unit deposits: Application to a porphyry copper deposit ${ }^{1}$
}

\author{
Serge Antoine Séguret ${ }^{2}$
}

\begin{abstract}
This work concerns mineral deposits consisting of geological bodies whose metal grades have different characteristics in terms of distribution and variogram which means that estimating grades by ordinary kriging may produce unrealistic spatial continuity. This paper proposes a method based on the indicators of the geological objects (hereafter called units) and their product with the metal grade. This is illustrated by an application to a porphyry copper deposit. The aim of this paper is essentially to promote the use of variogram ratios to analyze and characterize deposits.
\end{abstract}

Original version of a paper published by Mathematical Geosciences

DOI 10.1007/s11004-013-9475-0

\footnotetext{
${ }^{1}$ Received 26 September 2012 ; accepted 1 june 2013

${ }^{2}$ Centre de Geosciences, Mines ParisTech, Fontainebleau, France

e-mail: serge.seguret@mines-paristech.fr
} 
KEY WORDS: block modeling, mine, transition probabilities, relationship schemes, partial grades cokriging, indicator residual

\section{Introduction}

Block modeling is the division of a deposit into equal-size blocks, each block being quantified by, for example, a metal grade estimate. It represents an important step in the mining process as it conditions excavation at the end of open-cast projects, extension of underground mines, and monthly and yearly metal-quantity prediction for the plant. When the deposit consists of units with their own grade range and spatial variability, estimates of block grades that neglect unit specifics may produce unrealistic spatial continuity. This paper analyzes some practices commonly used to try to solve this problem and shows that they assume links between the geological bodies, which must be verified. Using the border effect phenomena and transition analyses between random sets (Rivoirard 1994) in a new way, where the sets produced by indicator function are disjoint, leads to the concept of the partial grades method (Séguret 2011). The approach is illustrated by an application on a porphyry copper deposit composed of eight units. As a byproduct, the concept of 
preferential relationship schemes is proposed, a powerful tool for geologists and mining engineers. An appendix contains all the required mathematical developments around the concepts of transitions and border effects.

\section{Current practice}

\subsection{Description}

Let us consider a deposit divided into equal-size blocks $V$ and let $Z(V)$ represent the average grade of the metal contained in a block. The geology is characterized by $n$ units or facies labeled $i$ at each location $x$ where there is a point support measurement $Z(x)$.

The formulation of the current practices is

$$
Z(V)=\sum_{i=1}^{n} \frac{v_{i}}{V} \frac{Q\left(v_{i}\right)}{\rho v_{i}}=\sum_{i=1}^{n} p_{i} Z\left(v_{i}\right)
$$

where $v_{\mathrm{i}}$ is the volume of unit $i$ contained in $V, Q\left(v_{i}\right)$ the corresponding quantity of metal, $\rho$ the density of the rock (assumed, for simplification, to be the same for all the units), $p_{i}$ the volumetric proportion of unit $i$ in $V$ and $Z\left(v_{i}\right)$ the grade associated with $v_{\mathrm{i}}$.

The estimation of $Z(V)$ is conducted in two steps:

Step 1 For each unit $i$, estimate $p_{i}$ and $Z\left(v_{i}\right)$ separately

Step 2 Combine the estimations to obtain the result

$$
Z^{* \wedge}(V)=\sum_{i=1}^{n} p_{i}^{*} Z^{\wedge}\left(v_{i}\right)
$$

The signs $*$ and ${ }^{\wedge}$ denote various estimations.

The ways in which the proportions $p_{i}$ are calculated vary. 
Practice 1: for each unit $i$, the geologist interpolates the samples labeled $i$ by manual mapping and the geological objects produced in this way define, in each block $V$, the volume $v_{\mathrm{i}}$.

Practice 2: based on the samples, proportions are calculated at a large scale by moving averages and then interpolated by kriging at the scale of blocks $V$.

Practice 3: as many indicator functions as there are units are defined and an indicator kriging or cokriging is made. The results are probabilities for a block to belong to any one unit. These probabilities are interpreted as proportions.

The way the grade is estimated is almost unique: for each unit $i$, a kriging at the scale of block $V$ is made using only the samples labeled $i$.

\subsection{Analysis}

The above-mentioned approaches are questionable.

Practice 1: the way the geologist draws the units has a direct impact on the resulting proportions, thus on the grade of block $\mathrm{V}$ and its economical value.

Practice 2: one usually starts with a large-scale proportion obtained via an average and then follows it with a kriging interpolation. This is a sequence of estimation that cannot be optimal and is incoherent. If decision is made to use the kriging method, why precede it by an average? In fact this approach is used when there are not enough data to directly conduct a kriging or cokriging of the indicator functions, or when the indicator variograms do not reveal any spatial structure. 
Practice 3: this approach is often used when the deposit is complex and the units have preferential locations in space, with the consequence that the indicator functions are not stationary. Then, usually, are defined homogeneous domains inside which proportions are calculated and the model depends on the domains. This spatial partitioning introduces discontinuities between the estimated domains which have to be smoothed in a subsequent process, where, for example, a moving average is used along the frontiers separating the domains. Here again, there is a combination of an objective approach (indicator kriging) with a questionable subsequent process.

In the following, exponents $K$ and $C K$ represent, respectively, kriging and cokriging. Note how the problem is written in Eq. (1).

A sum: the optimal estimation of a sum of variables is not necessarily equal to the sum of optimal estimators of each variable. For kriging, the equality is true when the variograms of the variables are all proportional to the same variogram (intrinsic correlation) (Wackernagel 1995). For cokriging, equality is true when the samples used for each unit $i$ are the same.

A product: the optimal estimation of the product $p_{i} Z\left(v_{i}\right)$ is not necessarily equal to the product of estimations. Separating the estimations may be justified if the terms of the product are spatially independent but even in that case, the optimality is not necessarily reached.

Support effect: Kriging the grade associated with the unit $i$, over $v_{\mathrm{i}}$, is not kriging it over $V$. Unit i only makes up fraction of $V$, not the whole block. But the usual 
practice consists in estimating this grade at the scale of $V$, producing an estimate $Z_{i}(V)^{K}$. As kriging is a linear function of the support, the resulting error produced by replacing $Z\left(v_{i}\right)^{K}$ by $Z_{i}(V)^{K}$ is

$$
Z_{i}(V)^{K}-Z\left(v_{i}\right)^{K}=\frac{v_{i}^{C}}{V}\left(Z\left(v_{i}^{C}\right)^{K}-Z\left(v_{i}\right)^{K}\right)
$$

In this formula, $v_{i}{ }^{C}$ is the complementary of $v_{i}$ inside $V$. The magnitude of the bias is proportional to the size of $v_{i}$ relatively to $V$ and large when $v_{i}$ is small compared to $V$.

\subsection{Questions}

If the proportions $p_{i}$ and the grades $\mathrm{Z}\left(v_{i}\right)$ are spatially correlated, might it be useful to use cokriging?

Is it possible to avoid direct proportions estimation during the block estimation procedure?

Why separate $p_{i}$ from $Z\left(v_{i}\right)$ ? Why not estimate their product directly, and what is the meaning of this product?

\section{Model}

\subsection{Construction}

For each unit $i$ among the $n$ units at our disposal and each point-support sample $x$, an indicator function $1_{i}(x)$ is defined

$$
\forall x, i, 1_{i}(x)=\mid \begin{array}{cc}
1 & \text { if } x \in \text { unit } i \\
0 & \text { otherwise }
\end{array} .
$$

As $x$ belongs to just one unit, for each location $x$ where there is a measurement, the sum of the indicator functions is 1 


$$
\sum_{\mathrm{i}=1}^{\mathrm{n}} 1_{\mathrm{i}}(x)=1 \forall x
$$

When this sum is multiplied by the grade $Z(x)$, which is the ratio of the metal mass divided by the sample tonnage assumed to be the same everywhere, the grade does not change

$$
Z(x)=Z(x) \sum_{\mathrm{i}=1}^{\mathrm{n}} 1_{\mathrm{i}}(x) \forall x
$$

But, by inverting the sum sign, appear products of the grade by the indicators

$$
Z(x)=\sum_{\mathrm{i}=1}^{\mathrm{n}} Z(x) 1_{\mathrm{i}}(x) \forall x .
$$

These products define the partial grades $Z_{i}(x)$

$$
Z_{i}(x)=Z(x) 1_{\mathrm{i}}(x) \forall x
$$

\subsection{Properties}

\subsubsection{Isotopic cokriging}

By this approach, the problem is transposed to an isotopic situation and the optimal estimation of the grade at the scale of a production block $V$ by cokriging based on the partial grades is equal to a sequence of partial-grade cokrigings

$$
Z(\mathrm{~V})^{\mathrm{CK}}=\sum_{\mathrm{i}=1}^{\mathrm{n}} Z_{\mathrm{i}}(\mathrm{V})^{\mathrm{CK}}
$$

\subsubsection{Metal versus tonnage}

In order to estimate a given partial grade $i$, the $n$ partial grades at our disposal are used, but also the $n-1$ indicators that support them ( $n-1$ and not $n$ because of relation (3)). In this way, both the metal variations inside the units and the geometry of the units are taken into account. 


\subsubsection{Probabilistic interpretation}

Handling indicators and partial grades in a multivariable context makes possible a novel use of tools developed by Jacques Rivoirard in the nineties: the probabilistic interpretation of the direct and cross indicator variograms and their ratios (see Appendix). The cross variogram between indicators $i$ and $j$ divided by the variogram of $i$ indicator quantifies the probability of encountering unit $j$ when leaving $i$

$$
\left|\frac{\gamma_{i j}(h)}{\gamma_{i}(h)}\right|=p(x+h \in j \mid x \in i, x+h \notin i) .
$$

The cross variogram between indicator $i$ and its partial grade $Z_{i}$ divided by the $i$ indicator variogram is simply the way the average grade increases or decreases with moves inside the unit

$$
\left|\frac{\gamma_{\mathrm{iz}_{\mathrm{i}}}(\mathrm{h})}{\gamma_{\mathrm{i}}(\mathrm{h})}\right|=\mathrm{E}[\mathrm{Z}(\mathrm{x}+\mathrm{h}) \mid \mathrm{x}+\mathrm{h} \in \mathrm{i}, \mathrm{x} \notin \mathrm{i}]
$$

\subsection{Procedure}

There are three steps:

Step 1 Geometry of the units

Using formula (6) makes possible detecting and quantifying the preferential contacts and shows how the transitions depend on distance. In the end is defined the list of indicators which are spatially linked and must be estimated together as auxiliary variables in the cokriging system, defined by (5).

Step 2 Metal variations inside units

Formula (7) makes possible detecting if metal variations exist and are large compared to the behavior of the unit geometries. If this is the case, the cokriging 
system (5) is used directly (possibly with indicator functions as auxiliary variables); if not, resorts the simplifications where the partial grades are expressed as a linear function of indicators plus residuals.

Step 3 The direct and cross variograms are modeled and the estimation is made.

\section{Application}

\subsection{Data}

The method is applied to a porphyry copper deposit in Chile. Its extent is approximately Easting 1000 meters, Northing 3000 meters and it is 1500 meters thick (Fig. 1). There are more than 100000 samples, to which a copper grade is assigned, and classified into eight units, according to the weathering of the rocks. Each unit presents a particular grade range (Fig. 2(a)) and has its own spatial variability (Fig. 2(b)).

\subsection{Geometry of the units}

Variogram ratios as defined by (6) make it possible to separate the units in contact with each other from those that are not and distinguish those with large contact zones. Figure 3(a) shows a transition between grey and brown, $3 \mathrm{~b}$ between brown and pink while $3 \mathrm{c}$ shows no (or minor) spatial transition between grey and pink. As brown acts as an intermediary between grey and pink, the three units, grey, brown and pink, are spatially linked and become useful together in a cokriging system. Figure 3(d) represents a clear transition which depends on the direction. In this deposit, the moment is reached when all the indicator functions are mutually informative, spatially correlated and a cokriging 
system is useful for estimating the proportions instead of estimating each proportion separately by kriging.

\subsection{Preferential relationship schemes}

The idea is to detect general laws in the mutual behavior of the units while analyzing transitions between all the pairs $(i, j)$. Therefore, formula (6) is calculated in the three main directions and are retained the pairs with positive Preferentiality Values $\operatorname{Pref}(i \rightarrow j)$ as defined by (19) in the Appendix. Results for the present case study (8 units) are in Table 1. The aim is detecting preferential contacts, i.e. only positive Preferentiality Values, and finding the dominant behaviors, so the results of Table 1 are classified into four classes and schemes are drawn up for each direction showing the relationships (Figs. 4 and 5). An arrow represents a spatial transition, and its color the magnitude of the transition. As these schemes are not connected with any location in space, the place of the colors can change, the scheme can turn by any degree, etc., and the only rule is to preserve the arrow and its color. If the presence, or not, of spatial transition and the ability to have a mutual transition, provide enough information to simplify an indicator cokriging system as mentioned above, such schemes are well understood by geologists and mining engineers. See Fig. 5. This scheme shows two subsets of units separated by grey. Geologists connected the scheme to the real space; the left set is the Western part of the deposit, the one on the right corresponds to the East. At an early stage of the feasibility study of this deposit, some years ago, geologists called the grey the "transition zone". This shows how these schemes "speak" to geologists.

Mining engineers linked these subsets to the grade ranges that appear in the figure, the cutoff value is the value of the grade below which it is not economically viable. Their 
conclusion is that, even if the left set is a great mixture where it is difficult to localize particular units, they are all above the cutoff value and discrimination is not a critical problem. This is not true for the right subset which contains a mixture of pink (rich) and brown (poor) and there, localizing brown and pink, and correctly quantifying their tonnage is critical. Before knowing these results, our industrial partner almost decided to stop mining in the grey Eastern part. If a method could improve the spatial discrimination of the units, they might reconsider their choice.

\subsection{Metal variations.}

Variogram ratios, as defined by (7) and detailed in the appendix, make it possible to quantify the enrichment or impoverishment when moving inside the unit. Figures 6(a) and 6(b) present the highest grade variations: $0.3 \%$ for yellow after $200 \mathrm{~m}, 0.5 \%$ for dark blue after $100 \mathrm{~m}$. These variations can be neglected when compared to the sills of the ratios, equal to $m_{/ i}$, the average only of the grades labeled $i$. For the other units, for example, light blue (Fig. 6(c)) and brown (Fig. 6(d)) there is no variation. Thus a model comes where the partial grade is expressed as a linear function of its indicator plus a residual, spatially independent from the indicator

$$
Z_{i}(x)=m_{/ i} 1_{i}(x)+R_{z_{i}}(x)
$$

In this expression, the slope of the regression $m_{/ i}$ can also be defined as the average of the partial grade $Z_{i}(x)$ divided by the $i$ unit proportion. Equation (8) produces as many residuals as units, their spatial correlation with each other and with the indicator functions is analyzed; the conclusion is that they are all spatially independent; thus the cokriging system (5) becomes a sequence with a cokriging of each unit proportion plus an individual kriging using only the residuals of the $i$ unit 


$$
\mathrm{Z}(\mathrm{V})^{\mathrm{CK}}=\sum_{\mathrm{i}=1}^{\mathrm{n}} m_{/ i} 1_{i}(\mathrm{~V})^{\mathrm{CK}}+\mathrm{R}_{i}(\mathrm{~V})^{\mathrm{K}}
$$

\subsection{Evaluation of the method}

Equation (9) shows that a preliminary calculation consists in estimating, at the scale of block $V$, the proportion of unit $i$, but this proportion estimation can also be done at the scale of the point support sample. A subset of around $10 \%$ of the data is built to avoid clusters and to achieve homogeneity in space. Then, for each unit and each selected sample (obviously hidden during the estimation), the cokriging of proportion is conducted, using the data of the subset and taking as unit estimate, the unit with the largest proportion estimation. In this way the practice of geologists is reproduced, consisting in allowing to the sample the unit that occupies the largest part of it. Of around 14,000 samples, the success rate is $79.4 \%$. Table 2 shows that unit 3092 gives the worst results $(57.2 \%$ success), the best being unit 305 (90.1\% success). These differences are probably linked to the spatial distribution of the units.

When the estimation of the grade this time is conducted, the correlation with truth is 0.635 for kriging and 0.643 for partial grade cokriging (Fig. 7), with a standard deviation of the error of around 0.27 .

Results are close for three reasons:

1. Insufficient contrast between the grades averages;

2. Ranges of the residual variograms too close to the range of the indicator variograms;

3. Large domains of the studied area contain just one or two units. 
Recall that the analyses lead to a model where each partial grade is expressed as a linear function of the indicator plus a residual which is spatially independent from any indicator and other residual (Eq. 8).

To prove the first assertion, the averages $m_{/ i}$ of Eq. (8) are changed according to Table 3, producing a realization of a deposit where there are more contrasts between the grades. It is realistic because we keep the geometry of the units and the natural fluctuation of the residuals; the new averages are plausible for a metallic deposit. A new cross validation is conducted with models based on the new data.

To prove the second assertion, independent residuals with no spatial structure (nugget effect i.e. white noise) are simulated while increasing the contrasts between the variances of the residuals (Table 4). A second cross validation is performed with models based on the new data.

To understand the third assertion, consider again Fig. 1: a compact domain of unit 305 (green) is located East of the deposit and the North domain consists essentially of unit 318 (brown) with very little 303 (grey) and some green. In these areas, there cannot be any great differences between kriging and cokriging as the moving neighborhood, a 200m wide window, captures only samples belonging to one or two units at a time. Consequently the performance of the methods is evaluated on a sub domain where many units intervene at the scale of the neighborhood (rectangles in Fig. 8).

Figure 9 shows the scatter diagram with the true values obtained on the complete domain when only the averages are changed and Fig. 10 shows the scatter diagrams with the true values on the restricted area and when the residuals have no spatial correlations inside each unit. Table 5 summarizes the results. Note that the gain in terms of correlation can 
reach 0.04 (0.69 for kriging, 0.73 for cokriging) which yields a reduction of $6 \%$ of the error interval. The performances are always better in the sub domain where many units intervene at the small scale as well as when the averages of the grades are different and better too when the spatial correlation of the grades inside the units disappears, the reason being that it increases the nugget effect of the variogram used for the usual kriging.

\section{Conclusions}

Analysis of usual practices shows that estimating the average grade $Z(V)$ does not require a priori a first estimate of unit proportions. The calculation can be made once using the cokriging of partial grades, a method which makes it possible to incorporate the unit specificities coherently in a single system. The application to a complex porphyry copper deposit where true unit codes are compared to their estimation, results in a success rate of $80 \%$. The same test applied to the grades shows some improvement compared to usual kriging, depending on the contrasts between the partial grades and the spatial behavior of the grades inside each unit. Analysis is easier in this deposit because the sensitive parameter is first and foremost the geometry of the units, and as a distant second, the behavior of the grades inside the units. It would be interesting to test the approach in a deposit that presents important border effects, a situation that has not been encountered neither on the three porphyry copper deposits, where this method has been applied so far, nor on a zinc deposit in Peru.

This work aims mainly to promote the use of variogram ratios to analyze and characterize deposits, producing a hierarchy between the unit geometry and the grade variations inside 
the units. A byproduct is the relationship scheme, a new powerful tool for geologists and mining engineers.

\section{Acknowledgments}

This work was initiated by Pedro Carrasco, and I sincerely hope that it may honor the memory of this eminent colleague and great friend. It has been fully supported by Codelco, Chile, and the French government I personally would like to thank them for their support, as well as two anonymous reviewers, the editor in chief and a professional translator who greatly contributed to improving the quality of the manuscript. 


\section{Appendix: Spatial transitions and border effects}

\section{Preliminary remarks}

The geological units (or facies) are interpreted as realizations of random sets (Matheron, 1975).

The same notation $i$ is used for unit indices and the sets themselves.

It is important to distinguish between the independency of two random sets $i$ and $j$, where in particular

$$
P(x \in i, x \in j)=P(x \in i) P(x \in j) .
$$

And disjoined sets defined by

$$
i \neq j, i \cap j=\varnothing \Leftrightarrow \forall x, 1_{i}(x) 1_{j}(x)=0 .
$$

Equation (11) expresses a spatial link between the sets making disjoined sets dependent and Eq. (10) false.

In the following $i$ and $j$ are disjoined sets.

\section{Spatial transitions}

As indicator function $l_{i}(x)$ of the unit $i$ is defined by Eq. (2), its mathematical expectation is the probability for $x$ to belong to $i$. When the indicator function $l_{i}(x)$ is sampled in $n$ locations, its mathematical expectation $p_{i}$, a probability, is interpreted as a spatial unit

proportion and approximated by $\frac{n_{i}}{n},\left(n_{i}\right.$ is the number of samples coded $\left.i\right)$.

The variances of $i$ indicator is limited by 0.25 (Fig. 11(a)) 


$$
\operatorname{Var}\left[1_{i}(x)\right]=p_{i}\left(1-p_{i}\right) \leq 0.25
$$

The indicator variogram $\gamma_{i}(h)$ is defined as in Serra (1982) by the variance of indicator increments and equals $0.5[P(x+h \in i, x \notin i)+P(x+h \notin i, x \in i)]$. Assuming the symmetry in $h$ of the probabilities leads to

$$
\gamma_{i}(h)=P(x \in i, x+h \notin i)
$$

The indicator variogram must satisfy the triangular inequality (Matheron 1988)

$$
\gamma_{i}\left(h+h^{\prime}\right) \leq \gamma_{i}(h)+\gamma_{i}\left(h^{\prime}\right)
$$

As a consequence, $\gamma_{i}(h)$ behaving as $a|h|^{\alpha}$, implies $\alpha \leq 1$. $\alpha$ close to 1 corresponds to a unit which has regular boundaries (Fig. 11(b)), $\alpha$ largely below 1 indicates irregular boundaries (Fig. 11(c)).

If the indicator variogram is stationary, the variance (Eq. (12)) becomes its sill: for large distances $h$, the events $\{x \in i\}$ and $\{x+h \notin i\}$ become independent and the probability of the pair of events is the product of their probabilities

$$
\text { sill of } \gamma_{i}(h)=p_{i}\left(1-p_{i}\right)
$$

The indicator cross variogram $\gamma_{i j}(h)$, defined as $0.5 E\left[\left(1_{i}(x+h)-1_{i}(x)\right)\left(1_{j}(x+h)-1_{j}(x)\right)\right]$, developed with Eq. (11) and assuming the symmetry in $h$, yields

$$
\gamma_{i j}(h)=-E\left[1_{i}(x) 1_{j}(x+h)\right]=-P(x \in i, x+h \in j) .
$$

For $h$ close to 0 , the absolute value of the cross variogram gives the probability of direct contact between $i$ and $j$ and practically, represents the counting of pairs of adjacent samples $(i, j)$. 
If the cross variogram is stationary, the events $\{x \in i\}$ and $\{x+h \in j\}$ for $h>$ range become independent and the probability of the pair of events equals the product of their probabilities

$$
\text { sill of } \gamma_{i j}(h)=-p_{i} p_{j} \text {. }
$$

As $i$ and $j$ are disjoined, $\{x+h \in j\} \Rightarrow\{x+h \notin i\}$ when $i \neq j$ and we have

$$
\gamma_{i j}(h)=-P(x \in i, x+h \in j, x+h \notin i) .
$$

Taking the absolute value of Eq. (16) divided by Eq. (13) and by definition of the conditional probability, we have

$$
\left|\frac{\gamma_{i j}(h)}{\gamma_{i}(h)}\right|=P(x+h \in j \mid x \in i, x+h \notin i) .
$$

This is the probability of reaching $j$ when leaving $i$. In case of stationary direct and cross variograms, this ratio is bounded by a sill given by Eq. (15) divided by Eq. (14)

$$
\text { sill of }\left|\frac{\gamma_{i j}(h)}{\gamma_{i}(h)}\right|=\frac{p_{j}}{1-p_{i}} \text {. }
$$

This is the probability of belonging to $j$ related to what is not $i$.

How proceeding in practice? Using the samples, Eq. (17) is calculated and analyzed. Two quantities characterize the curve (Fig. 12)

1. $p(\rightarrow j \mid i \rightarrow)$, the value of the ratio for $h$ close to 0 which represents the probability of encountering $j$ while leaving $i$ (i.e., practically the counting of pairs of samples $(i, j)$ directly in contact divided by the total number of samples $i$ involved in the calculation), 
2. The sill of the curve, equal to $\frac{p_{j}}{1-p_{i}}$ when the deposit is stationary and large compared to the range.

As it is, $p(\rightarrow j \mid i \rightarrow)$ has no meaning for detecting preferential contacts because if the unit $j$ into which we enter is omnipresent in the domain, upon leaving any $i, j$ will be encountered often. However, it has to be compared to something. Can it be the proportion of $j$ ? Not exactly, because the contacts between $i$ and itself has no interest, so the reference is the proportion of $j$ relative to the proportion of what is not $i$ and this gives $\frac{p_{j}}{1-p_{i}}$, the sill of the ratio variogram. Finally, to quantify the preferential contacts, the interesting magnitude is the "Preferentiality value" from $i$ to $j$

$$
\operatorname{Pref}(i \rightarrow j)=P(\rightarrow j \mid i \rightarrow)-\frac{p_{j}}{1-p_{i}} .
$$

Preferential contact counting cannot be distinguished from the transition behavior. The physical interpretation is that if two units are more often in contact than they should be with regard to their proportions, their bodies share complementary shapes so their indicator functions are spatially linked.

There are four cases concerning the behavior of Eq. (17) along $h$ :

$p(\rightarrow j \mid i \rightarrow)=0$. The units $i$ and $j$ are never in direct contact, the contact starts at $h>h_{0}$

(Fig. 13(a)). Imagine that there are only 3 units $i, j$ and $k$. If $p(\rightarrow j \mid i \rightarrow)=0$, this is necessary because $k$ separates $i$ from $j$. There will be a preferential contact between $i$ and $k$, and between $j$ and $k$ and $h_{0}$ represents the minimum width of $k$ that has to be crossed in order to travel from $i$ to $j$. 
$\operatorname{Pref}(i \rightarrow j)<0$. The probability $p(\rightarrow j \mid i \rightarrow)$ is smaller than it should be regarding $p_{i}$ and $p_{j}$. (Fig. 13(b)). This case is similar to the previous one. The range of the ratio is linked to the average width of the units to be crossed before a return to the situation where $l_{i}(x)$ and $l_{j}(x+h)$ become spatially independent.

$\operatorname{Pref}(i \rightarrow j)=0$. The ratio is flat; there is no spatial transition and no preferential contact

(Fig. 13(c)). $\gamma_{i j}(h)$ is proportional to $\gamma_{i}(h)$. If the reverse situation is true $1_{i}(x)$ and $l_{j}(x)$ are in intrinsic correlation, and knowledge of one unit provides no information of the second one. If the reverse situation is false, the geometry of $j$ is subjected to the geometry of $i$, leading to an indicator residual model where indicator $j$ is expressed as a linear function of indicator $i$ plus a spatially independent residual.

$\operatorname{Pref}(i \rightarrow j)>0$. The contact probability decreases with the distance (Fig. 13(d)). Units $i$ and $j$ are preferentially in contact and it is the latter case that is exploited to build preferential relationship schemes.

Spatial transition analyses and the proportions involved in these calculations must be computed along directions to fit the anisotropies of the geological bodies and their preferential locations in space. Fig. 3(d) shows an example of directional dependency issued from the case study of this paper. North-South and vertical transitions are similar, while the transition is different in the West-East direction. Practically, the directional quantities $p(\rightarrow j \mid i \rightarrow)^{d i r}, p_{i}^{d i r}$ and $p_{j}^{d i r}$ are based only on the pairs of samples $(i, j)$ which respect to the directional constraint. 


\section{Border effects}

The cross variogram between the indicator of $i$ unit and its partial grade $Z_{i}(x)$ defined by Eq. (4) is

$$
\gamma_{i Z_{i}}(h)=\frac{1}{2} E\left[\left(1_{i}(x+h)-1_{i}(x)\right)\left(Z_{i}(x+h)-Z_{i}(x)\right)\right] .
$$

The quantity in square brackets is not null if $\{x+h \notin i, x \in i\}$ or $\{x+h \in i, x \notin i\}$ so we obtain

$\gamma_{i Z_{i}}(h)=\frac{1}{2} E[Z(x) \mid x+h \notin i, x \in i] P(x+h \notin i, x \in i)+\frac{1}{2} E[Z(x+h) \mid x+h \in i, x \notin i] P(x+h \in i, x \notin i)$ Assuming symmetry in $h$ and by Eq. (13) yields

$$
\gamma_{i Z_{i}}(h)=E[Z(x+h) \mid x+h \in i, x \notin i] \gamma_{i}(h) .
$$

So another ratio intervenes

$$
\frac{\gamma_{i Z_{i}}(h)}{\gamma_{i}(h)}=E[Z(x+h) \mid x+h \in i, x \notin i] .
$$

This ratio shows how the average grade increases or decreases when moving inside the $i$ unit. This property is named "border effect" by Rivoirard (1994). In the present study, but also in two other copper deposits (Séguret 2011), and a confidential zinc deposit in Peru, this ratio did not depend on $h, \gamma_{i Z_{i}}(h)$ is proportional to $\gamma_{i}(h)$, leading to a model where the partial grade is expressed as a linear function of the indicator function (Eq. 8).

\section{References}

Matheron G (1975) Random Sets and Integral Geometry. Wiley, New York 
Matheron G (1988) The internal consistency of models in geostatistics. In Armstrong M, ed. Proceedings of Geostatistics - The Third International Geostatistics Congress, Vol. I, Kluwer Academic, Dordrecht, pp 21-38

Rivoirard J (1994) Introduction to Disjunctive Kriging and Non-linear Geostatisics. Oxford University Press, Oxford

Séguret S A (2011) Block model in a multi facies context - Application to a porphyry copper deposit. In Beniscelli J, ed. Proceedings of Geomin2011- Second International Seminar on Geology for the Mining Industry, Gecamin, Santiago, Chile

Serra J (1982) Image Analysis and Mathematical Morphology. Academic Press, London Wackernagel H (1995) Multivariate Geostatistics. Springer-Verlag, Berlin 


\section{Figure captions}

Fig. 1 Two-dimensional projections of the data colored according to their unit (a) XoY, (b) YoZ, (c) XoZ

Fig. 2 (a) From top to bottom, histogram of the grade of units 305, 303 and 3091, (b) from top to bottom, variograms of the grade of units 318,312 and 301

Fig. 3 Cross indicator variogram divided by a single indicator variogram, as defined by (6) and interpreted as the probability to enter unit $i$ while leaving unit $j$. The calculations are made along the main directions Nord-South, East-West and Vertical. (a) and (b) show transitions while (c) has no (or very small) spatial transition. (d) shows transitions with large anisotropies.

Fig. 4 Preferential relationship schemes obtained after classification of the positive Preferentiality Values of Table 1. (a) Transitions in the North-South direction, (b) transitions along Vertical axis. The accompanying table indicates the values of the transitions.

Fig. 5 Preferential relationship scheme in the West-East direction. On the left, the units ranked by increasing grades. Upper left table recalls the coding of Preferentiality Values classification. 
Fig. 6 Cross variogram between indicator and partial grade divided by indicator variogram as defined by (7). It represents the way the grade decreases or increases while entering unit $i$. (a) and (b) show some transition, (c) and (d) its absence.

Fig. 7 Scatter diagrams between the truth and estimations. Grades are expressed in percentages. $\mathrm{Cu}$ is the true grade, $\mathrm{Cu}{ }^{K}$ its estimation by usual kriging, $\mathrm{Cu}{ }^{\mathrm{COK}}$ estimation by partial grades cokriging, rho is the correlation coefficient

Fig. 8 Two-dimensional projections of the data colored according to their unit. Rectangles are the sub domains where the units are mixed and new statistics calculated (a) XoY, (b) YoZ, (c) XoZ

Fig. 9 Scatter diagrams between the truth (contrasted grades) and estimations. Grades are expressed in percentages. $\mathrm{Cu}$ is the true grade, $\mathrm{Cu} u^{K}$ its estimation by usual kriging, $\mathrm{Cu}{ }^{\mathrm{COK}}$ estimation by partial grades cokriging, rho is the correlation coefficient

Fig. 10 Scatter diagrams between the truth (contrasted grades and white noise as residuals) and estimations on a sub domain where the units are mixed (rectangles of Fig. 8). Grades are expressed in percentages. $\mathrm{Cu}$ is the true grade, $\mathrm{Cu}{ }^{K}$ its estimation by usual kriging, $\mathrm{Cu}^{\mathrm{COK}}$ estimation by partial grades cokriging, rho is the correlation coefficient 
Fig. 11 (a) behavior of the sill of the indicator variogram as a function of the proportion $p_{i}$ (b) Typical random sets associated, for h close to 0 , to power indicator variograms with an exponent close to 1 (c) Typical random sets associated, for h close to 0 , to power indicator variograms with an exponent much lower than 1

Fig. 12 Practical inference of the parameters. The variogram ratio is calculated, as defined by (17). Behavior close to 0 represents the contact probability of encountering unit $j$ when leaving $i$, the sill (if any) represents the same probability but when the events $\{$ entering $j\}$ and $\{$ leaving $i$ \} are independent (assuming that the dimension of the deposit is large compared to the range of the ratio). The difference between the two quantities is the Preferentiality Value defined by (19)

Fig. 13 Different possible situations for the Preferentiality Values as defined by (19). (a) Units $i$ and $j$ are never in direct contact which starts at $h_{0}$, the minimum width of the bodies that have to be crossed to reach $j$ when leaving $i$. (b) Units $i$ and $j$ are in direct contact but less than they should be considering their proportions in the domain. (c) The Preferentiality Value is 0 , the transition does not depend on the distance, the cross variogram between $i$ and $j$ is proportional to the indicator variogram of unit $i$. (d) $i$ and $j$ have a preferential contact with regard to their proportions in the domain 


\begin{tabular}{|c|c|c|c|c|c|c|c|c|}
\hline From & 305 & 303 & 312 & 318 & 301 & 307 & 3092 & 3091 \\
\hline 305 & & $\begin{array}{l}0.42 \\
0.30 \\
0.39\end{array}$ & 0 & $\begin{array}{l}-0.17 \\
0.27 \\
-0.02\end{array}$ & $\begin{array}{l}0.02 \\
0.02 \\
0.02\end{array}$ & $\begin{array}{l}0.04 \\
0.10 \\
0.03\end{array}$ & 0 & 0 \\
\hline 303 & $\begin{array}{c}0.03 \\
-0.14 \\
0.08\end{array}$ & & 0 & $\begin{array}{c}0.04 \\
-0.04 \\
0.18\end{array}$ & $\begin{array}{r}0.01 \\
0.16 \\
-0.12\end{array}$ & $\begin{array}{l}0.03 \\
0.10 \\
0.10\end{array}$ & $\begin{array}{l}0.10 \\
0.18 \\
0.04\end{array}$ & $\begin{array}{l}0.06 \\
0.05 \\
0.03\end{array}$ \\
\hline 312 & 0 & $\begin{array}{c}0 \\
0 \\
0.02\end{array}$ & & 0 & $\begin{array}{l}0.46 \\
0.62 \\
0.84\end{array}$ & 0 & $\begin{array}{c}0 \\
0.16 \\
0\end{array}$ & $\begin{array}{l}-0.07 \\
0.22 \\
-0.13\end{array}$ \\
\hline 318 & $\begin{array}{c}0.07 \\
-0.04 \\
0.09\end{array}$ & $\begin{array}{l}0.56 \\
0.17 \\
0.23\end{array}$ & 0 & & 0 & $\begin{array}{l}0.13 \\
0.32 \\
0.18\end{array}$ & $\begin{array}{l}0.02 \\
0.03 \\
0.01\end{array}$ & 0 \\
\hline 301 & $\begin{array}{l}0.01 \\
0.01 \\
0.01\end{array}$ & $\begin{array}{l}0.09 \\
0.28 \\
0.04\end{array}$ & $\begin{array}{l}0.21 \\
0.17 \\
0.16\end{array}$ & 0 & & 0 & $\begin{array}{l}0.03 \\
0.14 \\
0.13\end{array}$ & $\begin{array}{l}-0.01 \\
0.36 \\
0.07\end{array}$ \\
\hline 307 & $\begin{array}{l}0.05 \\
0.07 \\
0.02\end{array}$ & $\begin{array}{l}-0.08 \\
-0.01 \\
-0.01\end{array}$ & 0 & $\begin{array}{l}0.45 \\
0.65 \\
0.39\end{array}$ & 0 & & $\begin{array}{l}0.05 \\
0.03 \\
0.03\end{array}$ & 0 \\
\hline 3092 & 0 & $\begin{array}{l}0.39 \\
0.48 \\
0.34\end{array}$ & $\begin{array}{c}0.02 \\
0.06 \\
0\end{array}$ & $\begin{array}{l}0.04 \\
0.06 \\
0.06\end{array}$ & $\begin{array}{l}0.03 \\
0.11 \\
0.06\end{array}$ & $\begin{array}{l}0.06 \\
0.03 \\
0.06\end{array}$ & & 0 \\
\hline 3091 & 0 & $\begin{array}{l}-0.02 \\
-0.02 \\
-0.02\end{array}$ & $\begin{array}{l}0.05 \\
0.09 \\
0.08\end{array}$ & 0 & $\begin{array}{l}0.31 \\
0.31 \\
0.75\end{array}$ & 0 & 0 & \\
\hline
\end{tabular}

Table 1 Preferentiality Values as defined by (Eq. 19). Each cell contains one value per direction, from top to bottom: N-S, W-E, Vertical 


\begin{tabular}{|c|c|c|c|c|}
\hline & Nb sample tested & Global \% & well estimated & Success \% \\
\hline All units & 14268 & 100 & 11334 & 79.4 \\
\hline $\mathbf{3 0 5}$ & 2459 & 17.2 & 2225 & 90.1 \\
\hline $\mathbf{3 1 8}$ & 3146 & 22 & 2449 & 77.8 \\
\hline $\mathbf{3 1 2}$ & 388 & 2.7 & 322 & 83 \\
\hline $\mathbf{3 0 3}$ & 3339 & 23.4 & 2519 & 75.4 \\
\hline $\mathbf{3 0 1}$ & 2120 & 14.9 & 1756 & 82.8 \\
\hline $\mathbf{3 0 7}$ & 1179 & 8.3 & 793 & 67.3 \\
\hline $\mathbf{3 0 9 2}$ & 468 & 3.3 & 268 & 57.2 \\
\hline $\mathbf{3 0 9 1}$ & 1169 & 8.2 & 1002 & 85.7 \\
\hline
\end{tabular}

Table 2 Evaluation of the unit estimate 


\begin{tabular}{|c|c|c|}
\hline & Natural grade mean $\boldsymbol{m}_{\boldsymbol{i}}$ by unit $(\boldsymbol{\%})$ & Contrasted grade mean $\boldsymbol{m}_{\boldsymbol{i}}$ by unit $(\boldsymbol{\%})$ \\
\hline Units $\mathbf{i}$ & & 0.1 \\
\hline $\mathbf{3 0 5}$ & 0.205 & 1 \\
\hline $\mathbf{3 1 8}$ & 0.460 & 2 \\
\hline $\mathbf{3 1 2}$ & 0.510 & 3 \\
\hline $\mathbf{3 0 3}$ & 0.670 & 4 \\
\hline $\mathbf{3 0 1}$ & 1.026 & 5 \\
\hline $\mathbf{3 0 7}$ & 1.106 & 6 \\
\hline $\mathbf{3 0 9 2}$ & 1.269 & 7 \\
\hline 3091 & 1.567 & \\
\hline
\end{tabular}

Table 3 Initial and modified grade means by unit 


\begin{tabular}{|c|l|c|}
\hline & \multicolumn{1}{|c|}{ Natural structure of the residuals } & Simulated structure of the residuals \\
\hline Unit & & \\
\hline $\mathbf{3 0 5}$ & 0.005 nugget effect & 0.04 nugget effect \\
\hline $\mathbf{3 1 8}$ & 0.017 nugget +0.0058 spherical $(400 \mathrm{~m})$ & 0.25 nugget effect \\
\hline $\mathbf{3 1 2}$ & 0.006 nugget effect & 0.47 nugget effect \\
\hline $\mathbf{3 0 3}$ & 0.034 nugget +0.019 spherical $(80 \mathrm{~m})$ & 0.81 nugget effect \\
\hline $\mathbf{3 0 1}$ & 0.076 nugget +0.085 spherical $(130 \mathrm{~m})$ & 1.21 nugget effect \\
\hline $\mathbf{3 0 7}$ & 0.017 nugget +0.011 spherical $(65 \mathrm{~m})+$ linear & 1.69 nugget effect \\
\hline $\mathbf{3 0 9 2}$ & 0.02 nugget +0.01 spherical $(300 \mathrm{~m})$ & 2.25 nugget effect \\
\hline $\mathbf{3 0 9 1}$ & 0.037 nugget +0.026 spherical $(65 \mathrm{~m})$ & 2.89 nugget effect \\
\hline
\end{tabular}

Table 4 Initial and modified structures of the residuals by unit 


\begin{tabular}{|c|c|c|c|c|c|c|c|}
\hline \multirow[b]{3}{*}{$\begin{array}{c}\text { Case } \\
\text { studies }\end{array}$} & \multirow[b]{3}{*}{ Specifics } & \multicolumn{6}{|c|}{ Correlation with the truth } \\
\hline & & \multicolumn{3}{|c|}{$\begin{array}{c}\text { (A) } \\
\text { Complete domain }\end{array}$} & \multicolumn{3}{|c|}{$\begin{array}{c}(\mathrm{B}) \\
\text { Restricted area }\end{array}$} \\
\hline & & Kriging & Cokriging & $\begin{array}{c}\text { Gain } \\
\Delta \rho\end{array}$ & Kriging & Cokriging & $\begin{array}{c}\text { Gain } \\
\Delta \rho\end{array}$ \\
\hline (1) & $\begin{array}{ll}\text { - } & \text { Natural } \\
\text { indicators } \\
\text { - } & \text { Natural averages } \\
\text { - } & \text { Natural residuals } \\
\end{array}$ & $\rho=0.635$ & $\rho=0.643$ & 0.008 & $\rho=0.614$ & $\rho=0.623$ & 0.009 \\
\hline (2) & $\begin{array}{ll}\text { - } & \text { Natural } \\
\text { indicators } \\
\text { - } & \text { Contrasted } \\
& \text { averages } \\
\text { - } & \text { Natural residuals } \\
\end{array}$ & $\rho=0.835$ & $\rho=0.849$ & 0.014 & $\rho=0.813$ & $\rho=0.834$ & 0.021 \\
\hline (3) & $\begin{array}{ll}\text { - } & \text { Natural } \\
\text { indicators } \\
\text { - } & \text { Contrasted } \\
& \text { averages } \\
\text { - } & \text { Noisy residuals } \\
\end{array}$ & $\rho=0.763$ & $\rho=0.780$ & 0.017 & $\rho=0.688$ & $\rho=0.727$ & 0.039 \\
\hline
\end{tabular}

Table 5 Correlations with the true values, comparison between usual kriging and partial grades cokriging. Application on three "deposits": (1) the initial one, (2) greater differences between the average grades by unit according to Table 3, (3) greater differences between the averages grades by unit according to Table 3 and noisy residuals according to Table 4. Statistics are established (A) all over the domain, and (B) over a restricted area where many units intervene at the neighborhood scale (rectangles of Fig. 8)

\section{Figure 1}



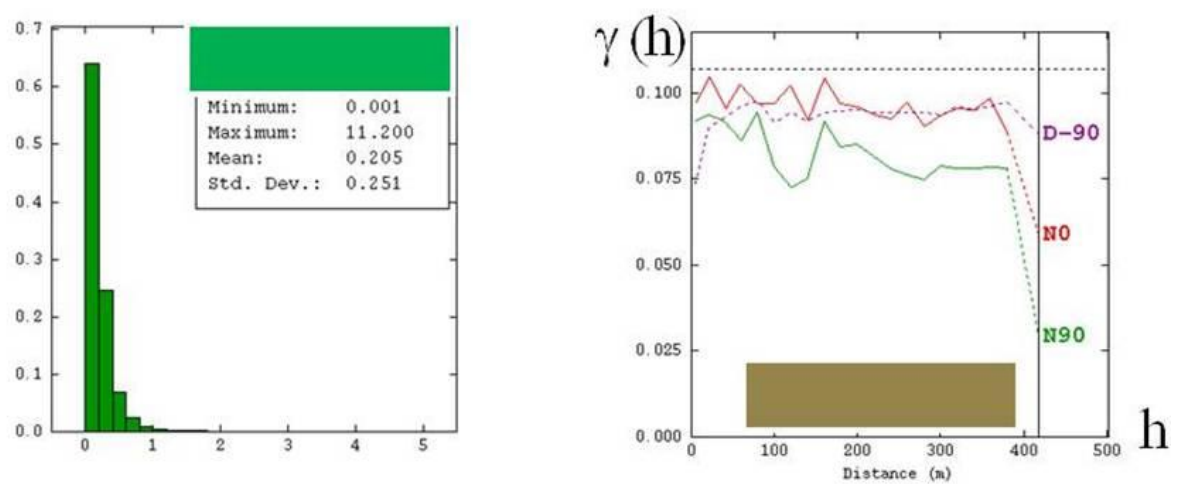

318

312
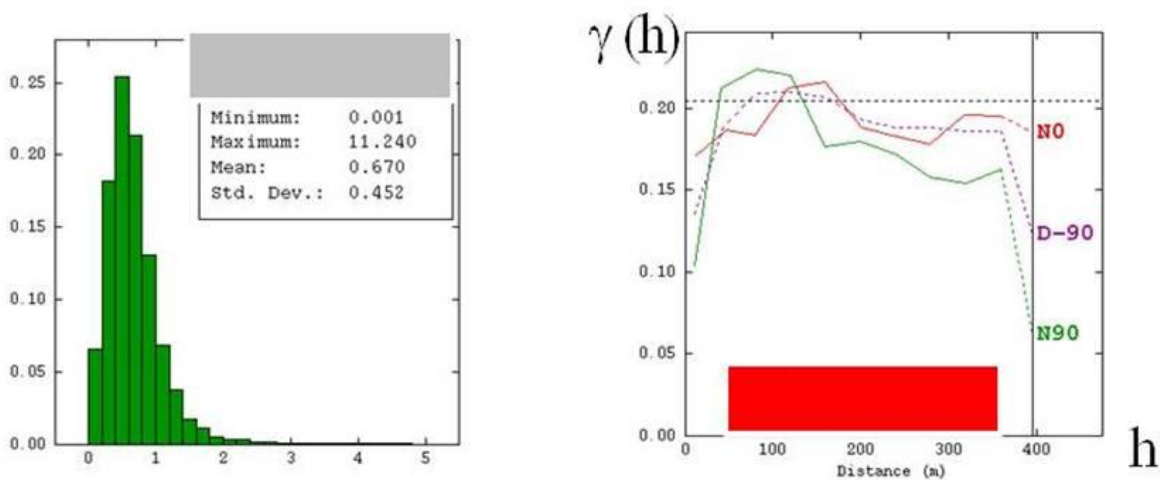

3091
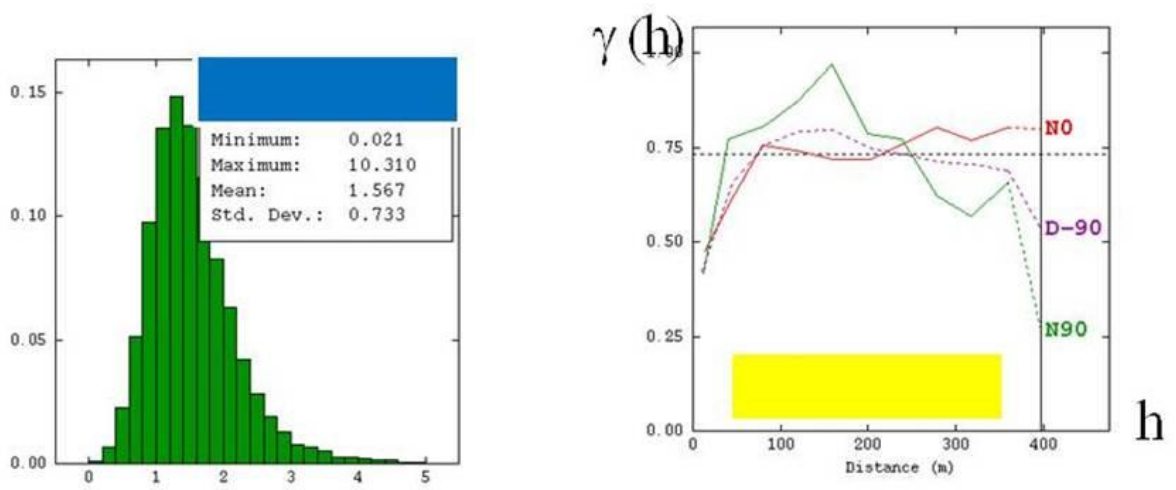

(a)

(b)

Figure 2 

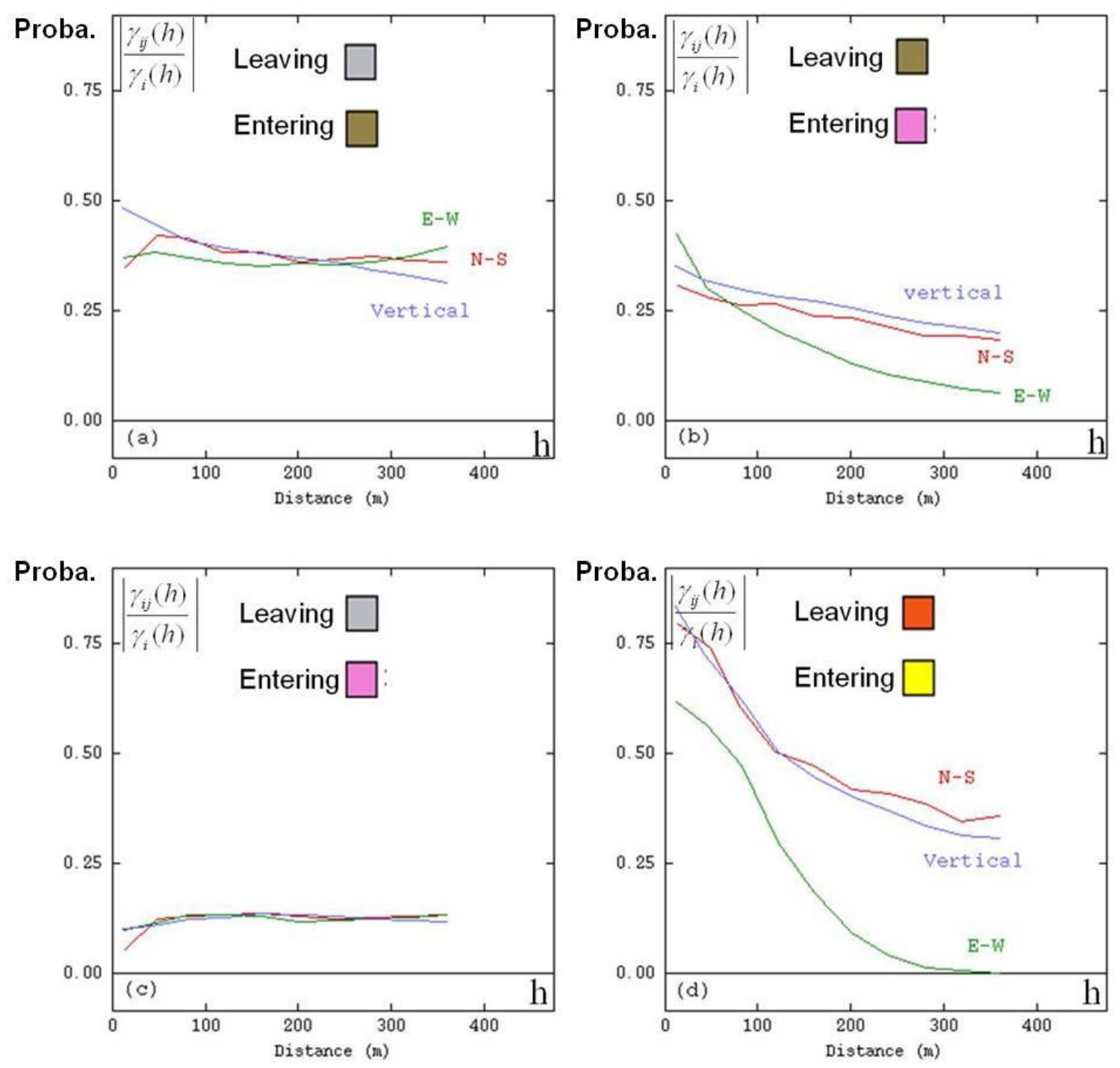

Figure 3 


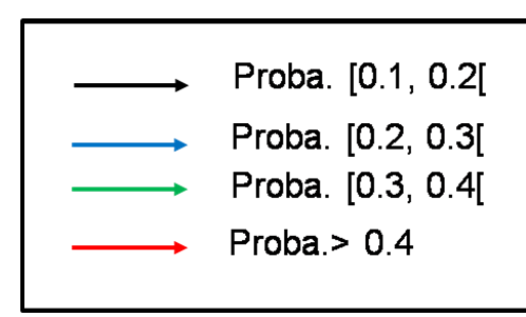

(a)

(b)

(b)

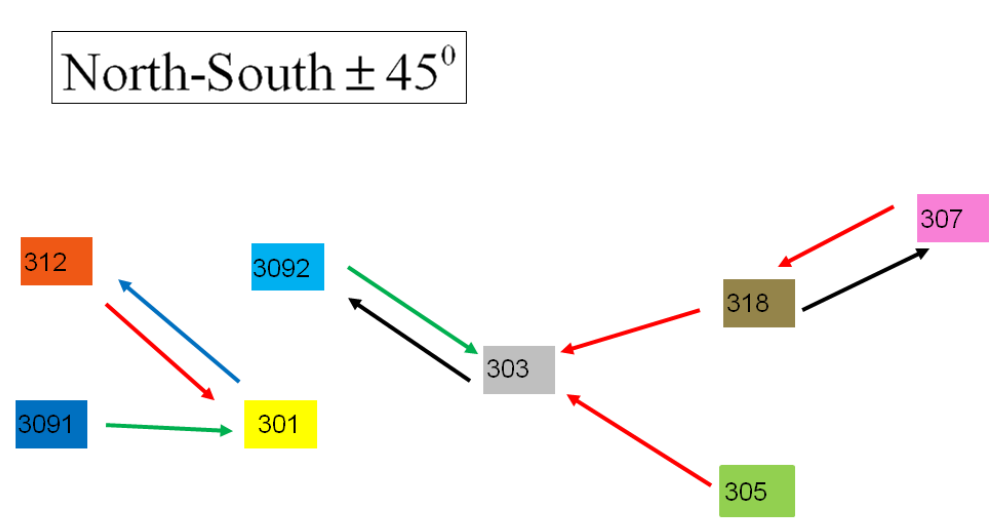

Vertical $\pm 45^{\circ}$

\section{Figure 4}




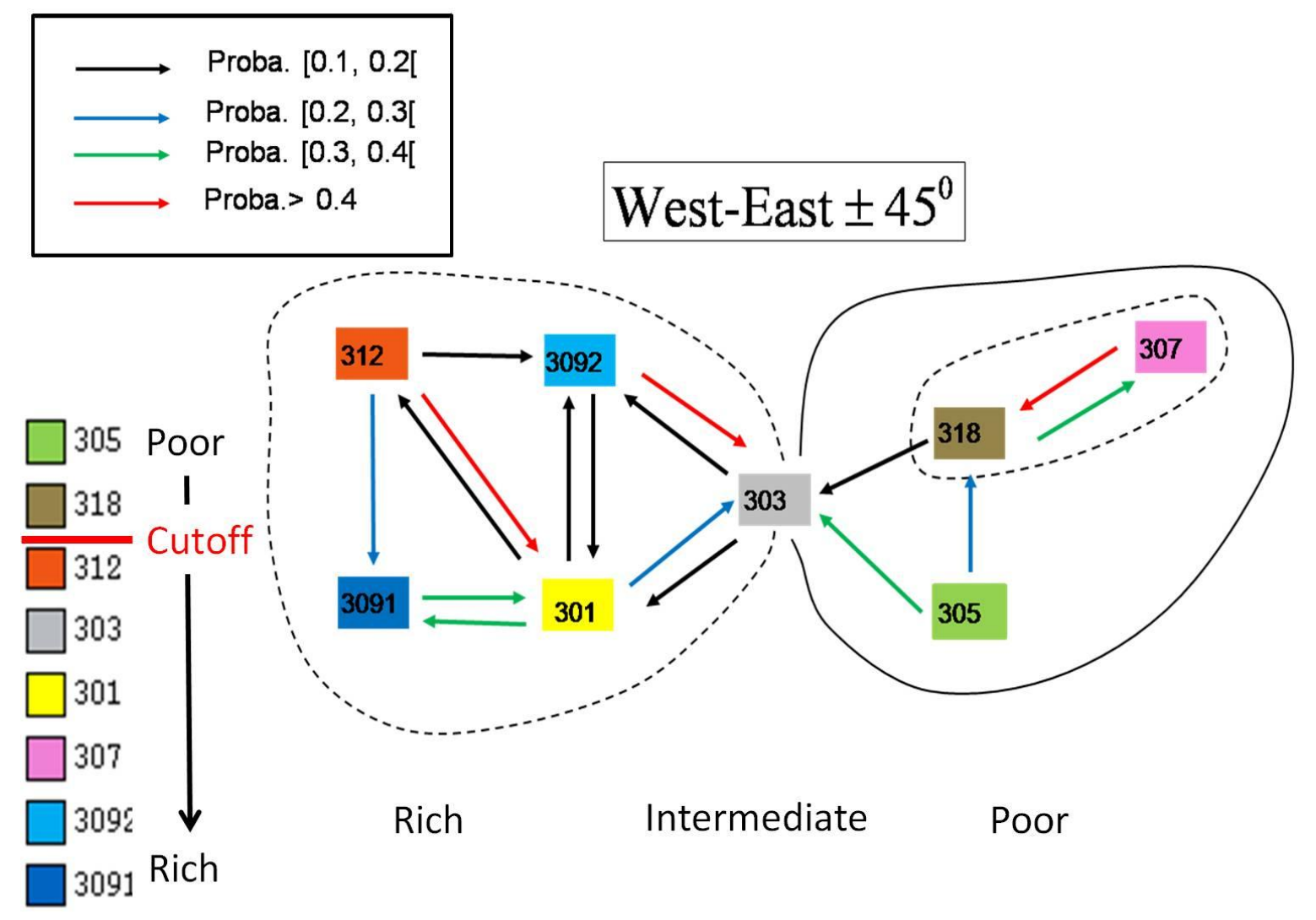

Figure 5 

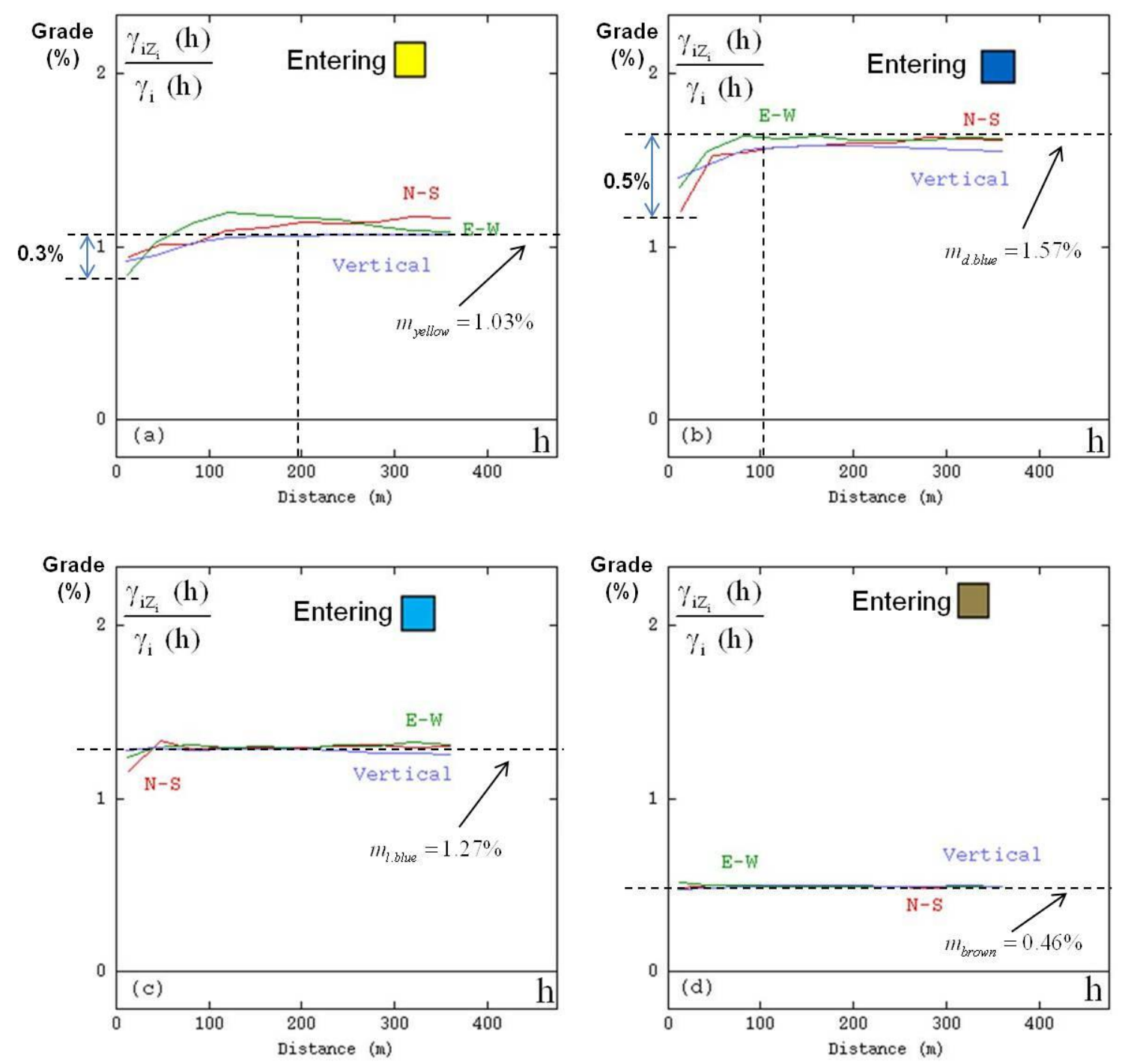

Figure 6 

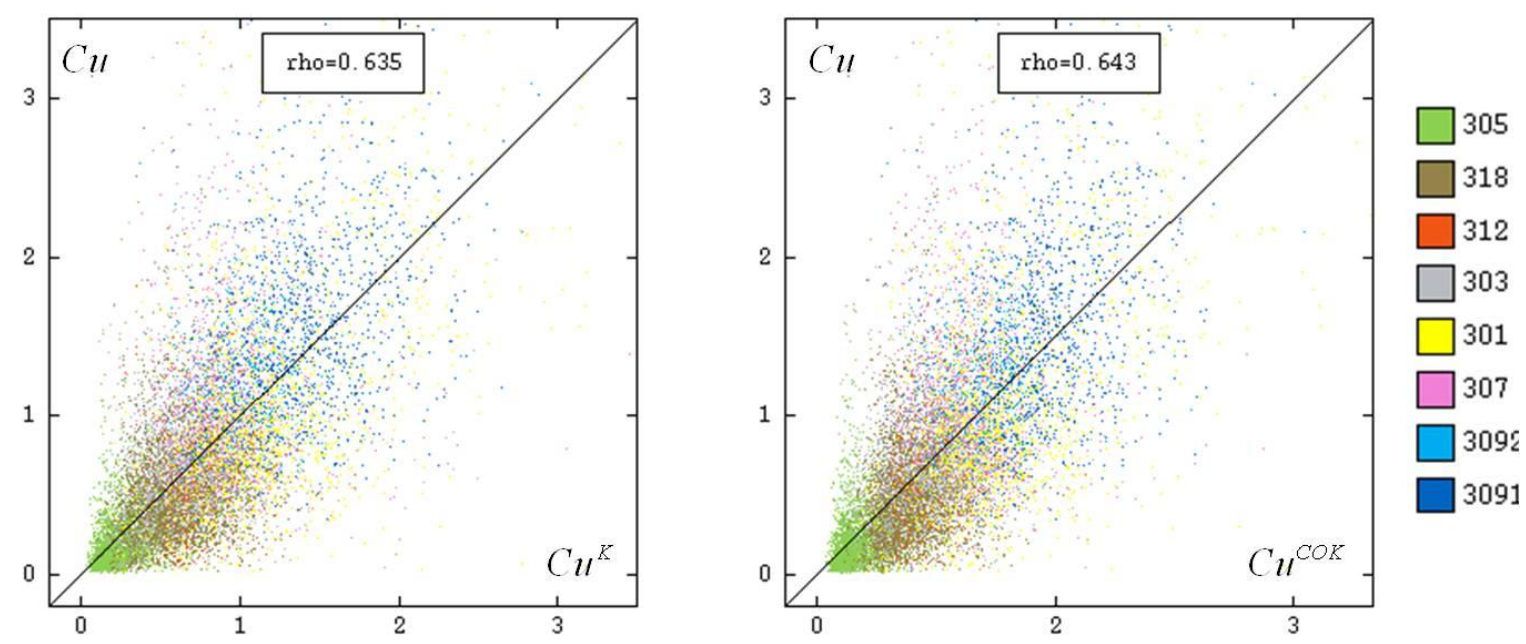

Figure 7 

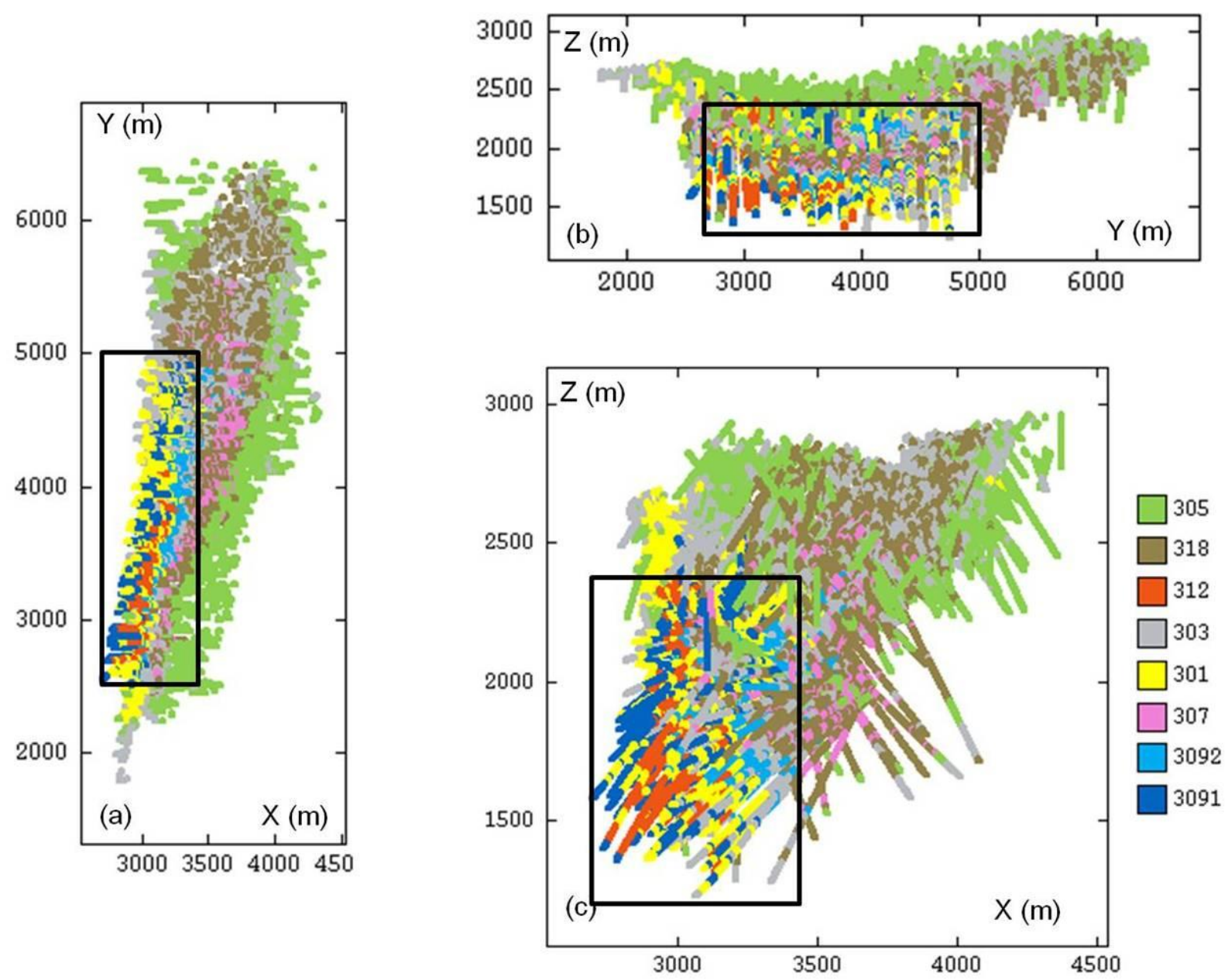

Figure 8 

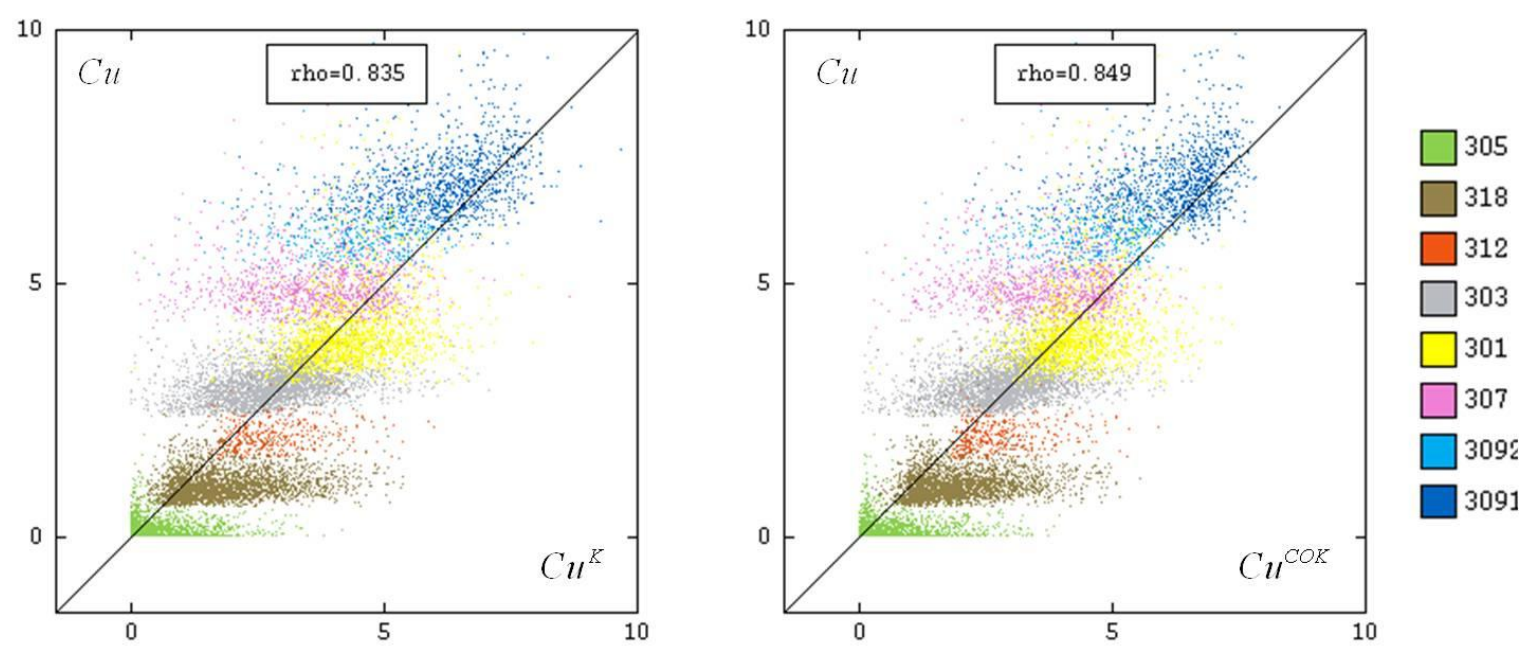

Figure 9 

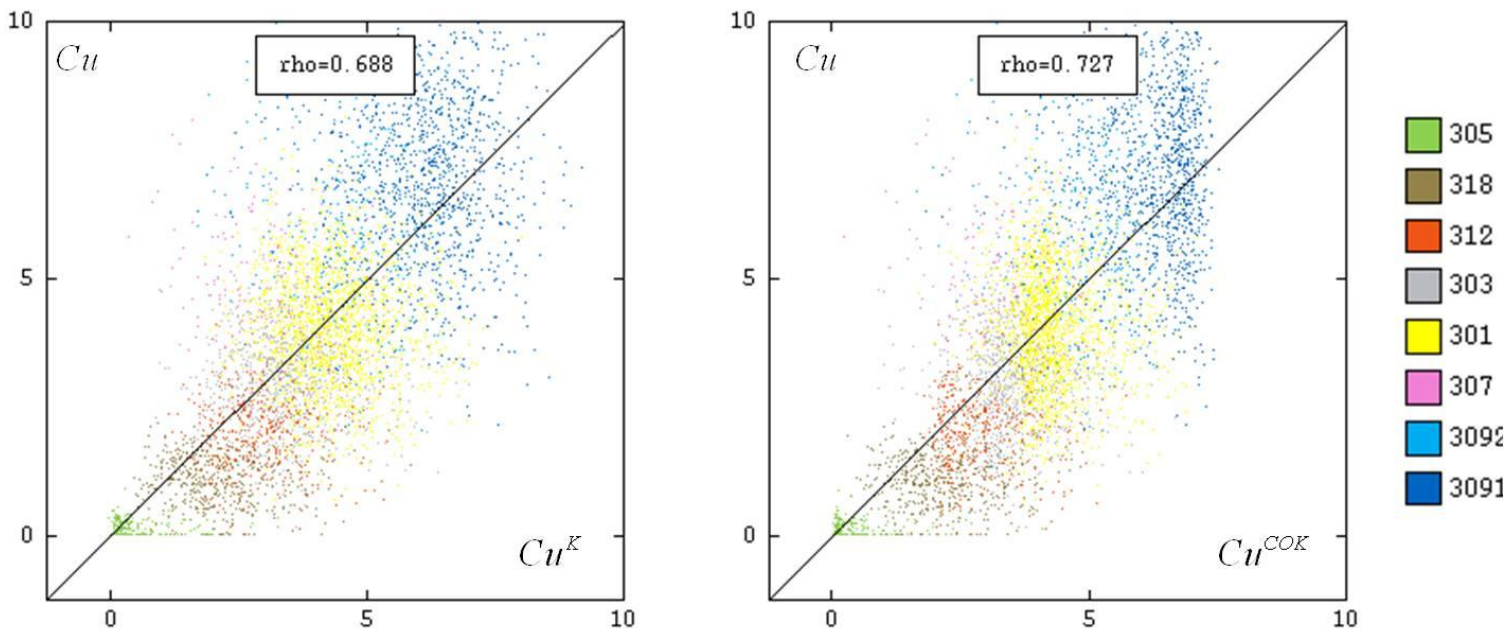

Figure 10 


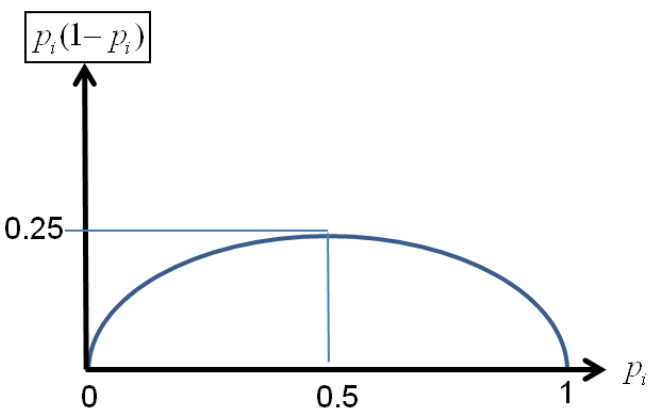

(a)

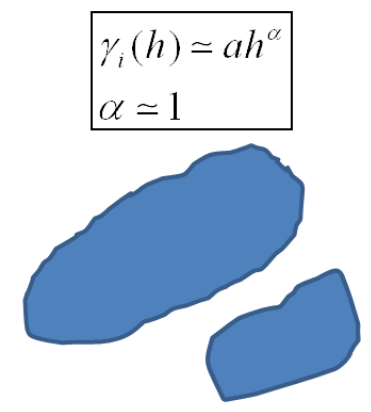

(b)

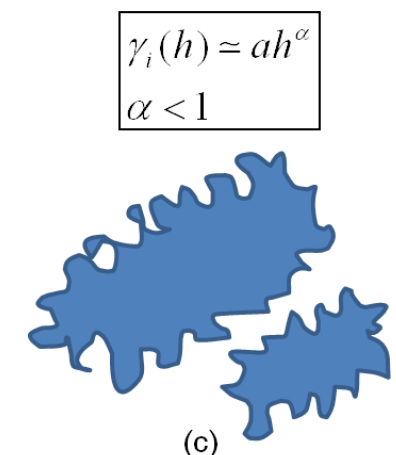

(c)

Figure 11 


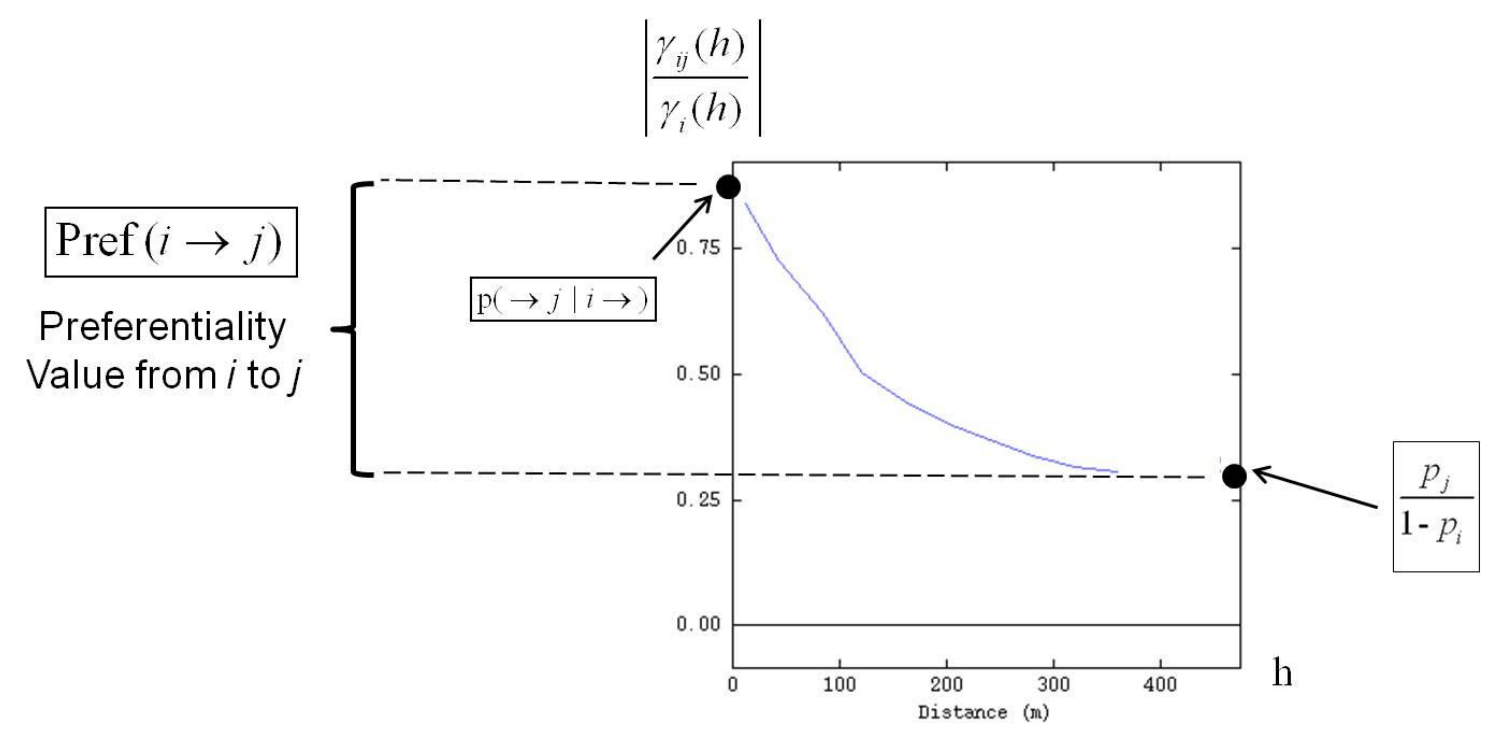

Figure 12 


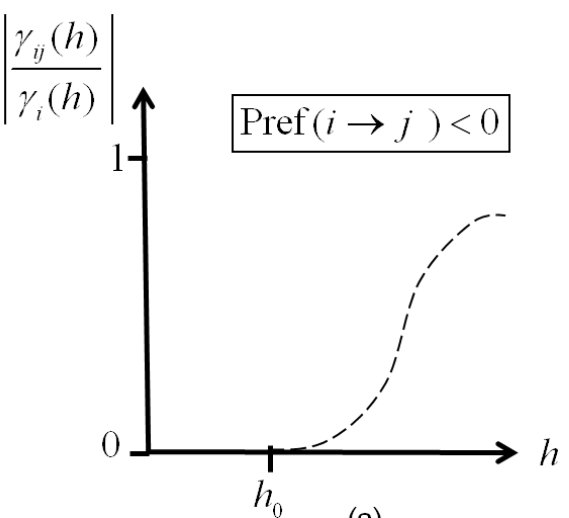

(a)

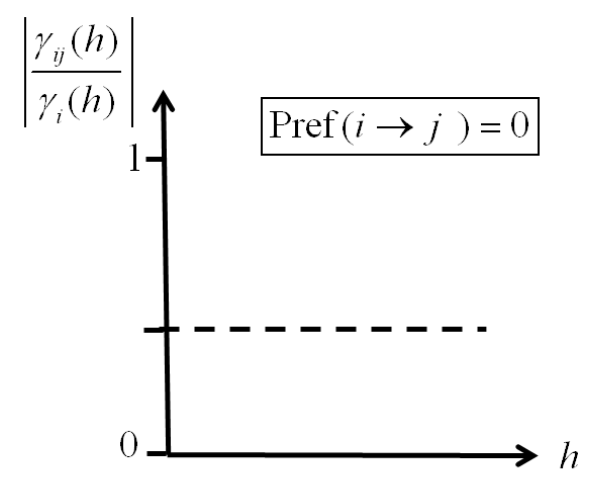

(c)

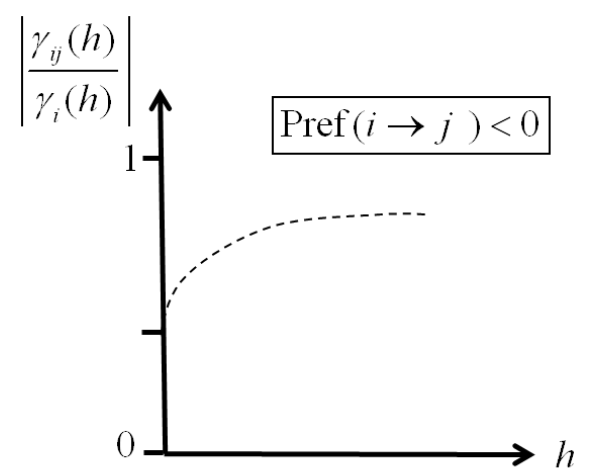

(b)

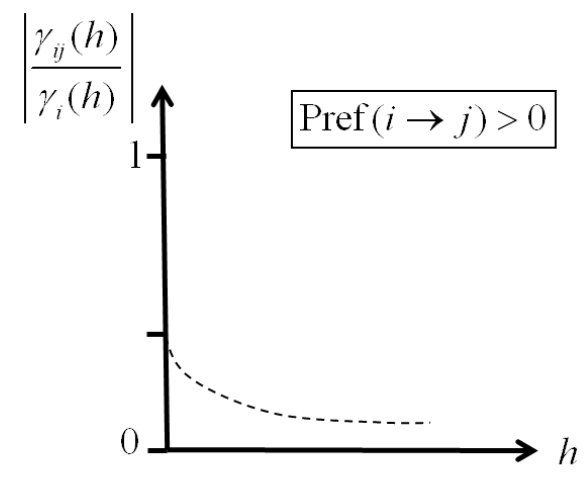

(d)

Figure 13 